\title{
Clinical Outcomes of Patients With Locally Advanced Rectal Cancer With Persistent Circumferential Resection Margin Invasion After Preoperative Chemoradiotherapy
}

\author{
Chang Hyun Kim ${ }^{1}$, Seung-Seop Yeom ${ }^{1}$, Hand-Duk Kwak², Soo Young Lee ${ }^{1}$, Jae Kyun Ju², Young Jin Kim, \\ Hyeong Rok Kim ${ }^{1}$ \\ ${ }^{1}$ Department of Surgery, Chonnam National University Hwasun Hospital and Medical School, Gwangju; ${ }^{2}$ Department of Surgery, Chonnam \\ National University Medical School, Gwangju, Korea
}

Purpose: Treatment after failure of circumferential resection margin (CRM) conversion after preoperative chemoradiotherapy (pCRT) for locally advanced rectal cancer (LARC) has not been evaluated well. We conducted a single-center, retrospective analysis to fill this information gap.

Methods: From 2008 to 2016, we included 112 patients who had predictive CRM involvement on baseline magnetic resonance imaging (MRI) and who underwent surgery following pCRT for LARC. Baseline and posttreatment radiologic and clinical factors were analyzed.

Results: Of 493 patients with LARC, 112 had CRM involvement by baseline MRI (mrCRM). In 40 patients (35.7\%), mrCRM involvement was converted as negative posttreatment CRM (ymrCRM-). Multivariate analysis showed the risk factors for persistent CRM involvement $(\mathrm{ymrCRM}+)$ after pCRT were extramural venous invasion $(\mathrm{mrEMVI}+)(\mathrm{P}=0.030)$ and lower tumor location $(\mathrm{P}=0.007)$. In addition, persistent CRM involvement after pCRT was an independent risk factor for predicting pathologic CRM involvement. The Cox proportional hazard model showed baseline positive mrEMVI remained significant for disease-free survival $(\mathrm{DFS})(\mathrm{P}<0.001)$. On posttreatment $\mathrm{MRI}$, abdominoperineal resection $(\mathrm{P}=$ 0.031), intersphincteric resection $(P=0.006)$, and persistent $C R M$ involvement $(P=0.001)$ remained significant for local recurrence-free survival. With regard to DFS, persistent CRM involvement $(\mathrm{P}=0.048)$ and positive EMVI on posttreatment MRI (ymrEMVI) ( $\mathrm{P}=0.014)$ were significant. In the patient subgroup with persistent CRM involvement, 5-year DFS in patients with mrEMVI and ymrEMVI was $29.8 \%$ and $21.2 \%$, respectively.

Conclusion: Patients who fail to convert to negative CRM have extremely poor oncologic outcomes. Lower tumor height and negative mrEMVI status were good responders to ymrCRM conversion. Our results suggest that these patients require a more intensive treatment modality.

Keywords: Rectal neoplasms; Chemoradiotherapy; Resection margin; Chemoradiotherapy; Prognostic factor

Received: February 11, 2019 - Accepted: April 22, 2019

Correspondence to: Hyeong Rok Kim, M.D.

Department of Surgery, Chonnam National University Hwasun Hospital and Medical School, 322 Seoyang-ro, Hwasun-gun 58128, Korea

Tel: +82-61-379-7646, Fax: +82-61-379-7661,E-mail: drkhr@jnu.ac.kr ORCID code: https://orcid.org/0000-0003-2737-0485

\section{(c) 2019 The Korean Society of Coloproctology}

This is an open-access article distributed under the terms of the Creative Commons Attribution NonCommercial License (http://creativecommons.org/licenses/by-nc/4.0) which permits unrestricted noncommercial use, distribution, and reproduction in any medium, provided the original work is properly cited.

\section{INTRODUCTION}

After introduction of total mesorectal excision (TME) and preoperative chemoradiotherapy (pCRT), the local recurrence rate after surgery for locally advanced rectal cancer (LARC) decreases significantly [1]. Over the last decade, continuous efforts have been made to use tailored treatment strategies for these patients. Although there are many efforts to preserve the rectum and to improve the functional outcome of LARC treatment $[2,3]$, knowledge of tumors that require more extensive surgical and/or oncologic treatment is limited.

In this regard, the most recent National Comprehensive Cancer 
Network (NCCN) guidelines recommend an additional 12 to 16 weeks of chemotherapy, if persistent circumferential resection margin (CRM) involvement or residual bulky tumor is revealed on restaging magnetic resonance imaging (MRI) after completion of pCRT. However, this strategy has not been supported by firm evidence and was only assessed in a small study with 45 patients [4]. Furthermore, few studies have evaluated the oncological outcome of patients with failed CRM conversion after pCRT for LARC, and the number of patients included was limited (38-88 patients) $[5,6]$.

In this study, we report the results for oncologic outcomes of patients who had CRM involvement on pretreatment MRI. This study aimed to identify the predictors of persistent CRM involvement despite the use of current standard long-course pCRT and pathologic CRM. Furthermore, we also assessed the prognostic relevance, as judged by local recurrence-free survival (LRFS) and disease-free survival (DFS), of posttreatment MRI parameters in patients with persistent CRM involvement.

\section{METHODS}

Prospectively collected data from 493 patients with rectal cancer who underwent surgery after pCRT at our tertiary care academic center between January 2009 and June 2016 were reviewed retrospectively. The study was approved by the Institutional Review Board of Chonnam National University Hwasun Hospital (TMP2019-85), which waived the requirement for informed consent in this retrospective study. Of 493 patients, 112 had CRM involvement based on pretreatment MRI. Patients with negative CRM involvement $(n=365)$ and metastatic disease $(n=5)$, those who refused to undergo surgery $(\mathrm{n}=2)$, those without baseline MRI ( $\mathrm{n}$ $=8)$, and those who underwent local excision $(\mathrm{n}=1)$ were excluded (Fig. 1). Pretreatment clinical evaluation included physical examination, rigid sigmoidoscopy, colonoscopy, abdominopelvic computed tomography (CT), rectal MRI, chest CT, and measurement of serum carcinoembryonic antigen (CEA).

All patients treated with pCRT underwent posttreatment MRI 4-6 weeks after completion of pCRT. Details of the pCRT have been previously described [7]. Radiotherapy consisted of 4,040$4,500 \mathrm{cGy}$ in 25-28 fractions delivered to the primary tumor and pelvic lymph node by using a 4 -field box technique. During the first and fifth weeks of radiotherapy, chemotherapy, consisting of 5 -fluorouracil $\left(425 \mathrm{mg} / \mathrm{m}^{2} /\right.$ day $)$ and leucovorin $\left(20 \mathrm{mg} / \mathrm{m}^{2} /\right.$ day $)$, was administered intravenously. Adjuvant chemotherapy was initiated 4-6 weeks postoperatively in patients who received preoperative or postoperative CRT. MRI was assessed by 2 radiologists and reviewed at a multidisciplinary team meeting. The distal edge of the rectal tumor was measured from the anal verge on the basis of rigid sigmoidoscopy, and tumors within $15 \mathrm{~cm}$ were included in the study. Low rectal cancer was defined as having low $(<5 \mathrm{~cm})$, $\operatorname{mid}(\leq 5$ and $<10 \mathrm{~cm}$ ), and upper borders $(\geq 10 \mathrm{~cm})$ from the anal verge. In addition, tumor size, lymph node involvement, CRM,

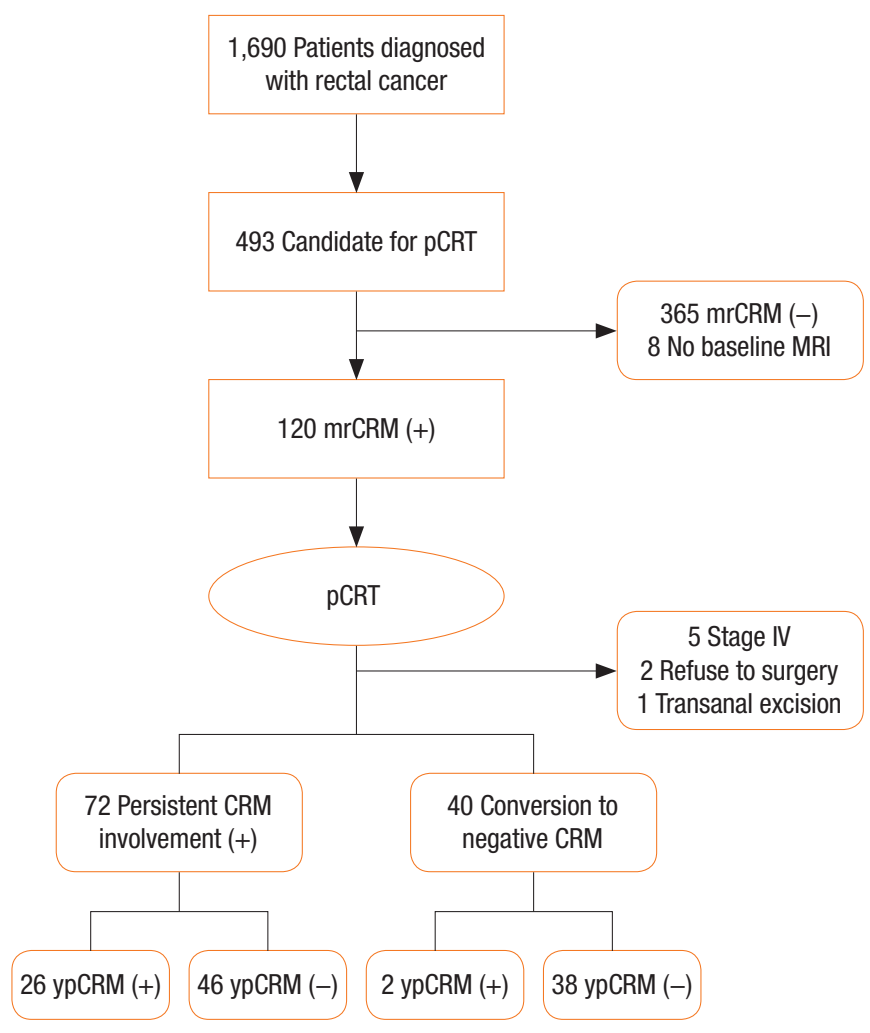

Fig. 1. Flow diagram. pCRT, preoperative chemoradiotherapy; mrCRM, circumferential resection margin based on pretreatment magnetic resonance imaging; ypCRM, pathologic circumferential resection margin.

and vascular invasion were measured using electronic calipers on a PACS system. Baseline MRI CRM (mrCRM) involvement was defined if the tumor distance to the mesorectal fascia was $<1 \mathrm{~mm}$ on MRI. Tumor regression grade by MRI (mrTRG) was defined as previously reported [8]. In this study, the 5-score system was simplified to a binary score (1/2 vs. $3 / 4 / 5)$.

Surgical options included high ligation of the inferior mesenteric artery, TME, or extended TME (TME with en bloc resection of the adjacent involved organ). The interval between preoperative CRT and surgery was 6-8 weeks. The patients underwent standardized follow-up at 3-month intervals for 2 years and at 6 -month intervals for the next 3 years. Local recurrence was defined as any recurrent disease in the pelvic cavity, including bowel anastomosis. Distant metastasis was defined as disease outside the pelvis.

The $\chi^{2}$ test or Fisher exact test was used to analyze the significance of categorical variables, and the Student $\mathrm{t}$-test was used for continuous variables. Univariate logistic regression analysis was used to identify the predictors of ymrCRM and pathologic CRM (ypCRM) in the pre- and posttreatment variables, respectively. Variables that were significant at $\mathrm{P}<0.10$ in the univariate analysis were considered in a backward stepwise multivariate logistic 
Table 1. Univariable and multivariable analysis of persistent circumferential resection margin (CRM) invasion on magnetic resonence imaging after preoperative chemoradiotherapy

\begin{tabular}{|c|c|c|c|c|c|c|}
\hline \multirow{2}{*}{ Variable } & \multicolumn{2}{|c|}{ Univariate analysis } & \multirow{2}{*}{ P-value } & \multicolumn{2}{|c|}{ Multivariate analysis } & \multirow{2}{*}{ P-value } \\
\hline & Persistent CRM $(n=72)$ & Converted CRM $(n=40)$ & & $\mathrm{OR}$ & $95 \% \mathrm{Cl}$ & \\
\hline Age (yr) & $64.0 \pm 11.0$ & $65.0 \pm 10.5$ & 0.617 & & & \\
\hline Sex & & & 1.000 & & & \\
\hline Male & $51(70.8)$ & $29(72.5)$ & & & & \\
\hline Female & 21 (29.2) & $11(27.5)$ & & & & \\
\hline Body mass index $\left(\mathrm{kg} / \mathrm{m}^{2}\right)$ & $22.6 \pm 3.1$ & $22.9 \pm 2.6$ & 0.602 & & & \\
\hline Hemoglobin (g/dL) & $12.7 \pm 2.3$ & $12.7 \pm 2.4$ & 0.925 & & & \\
\hline Tumor location & & & 0.055 & & & 0.007 \\
\hline Low & 17 (23.6) & $18(45.0)$ & & 1.000 & - & \\
\hline Mid/upper & $55(76.4)$ & $22(55.0)$ & & 3.927 & $1.492-11.182$ & \\
\hline Tumor size $(\mathrm{cm})$ & & & 0.977 & & & \\
\hline$\leq 5$ & $29(40.3)$ & $17(42.5)$ & & & & \\
\hline$>5$ & $43(59.7)$ & $23(57.5)$ & & & & \\
\hline CEA at diagnosis (ng/mL) & & & 0.122 & & & \\
\hline$\leq 5$ & $26(36.1)$ & $21(52.5)$ & & & & \\
\hline$>5$ & $46(63.9)$ & $19(47.5)$ & & & & \\
\hline mrT category & & & 0.020 & & & 0.268 \\
\hline $2 / 3$ & 47 (65.3) & 35 (87.5) & & 1.000 & - & \\
\hline 4 & $25(34.7)$ & $5(12.5)$ & & 1.936 & $0.628-6.771$ & \\
\hline mrN category & & & 0.458 & & & \\
\hline 0 & $6(8.3)$ & $7(17.5)$ & & & & \\
\hline 1 & 21 (29.2) & $10(25.0)$ & & & & \\
\hline 2 & $45(62.5)$ & $23(57.5)$ & & & & \\
\hline mrEMVI & & & $<0.001$ & & & 0.030 \\
\hline No & $8(11.1)$ & $16(40.0)$ & & 1.000 & - & \\
\hline Yes & $64(88.9)$ & $24(60.0)$ & & 3.319 & $1.146-10.149$ & \\
\hline Differentiation & & & 0.421 & & & \\
\hline Well & 38 (52.8) & 25 (62.5) & & & & \\
\hline Moderately & 31 (43.1) & 15 (37.5) & & & & \\
\hline Poorly & $3(4.2)$ & $0(0)$ & & & & \\
\hline Pathologic CRM & & & 0.436 & & & \\
\hline Involved & $26(36.1)$ & $2(5.0)$ & & & & \\
\hline Not involved & 46 (63.9) & 38 (95.0) & & & & \\
\hline
\end{tabular}

Values are presented as mean \pm standard deviation or number (\%).

$\mathrm{CEA}$, carcinoembryonic antigen; EMVI, extramural venous invasion; $\mathrm{OR}$, odds ratio; $\mathrm{Cl}$, confidence interval.

regression model. The Kaplan-Meier method was used to establish the effects of each variable, and log-rank tests were used to compare survival curves. Multivariate survival analyses were performed using Cox regression models with calculation of hazard ratios (HRs) and 95\% confidence intervals (CIs). These analyses were performed separately on the pretreatment variables (i.e.,
mrT, mrN, and mrEMVI), posttreatment variables (i.e., ymrT, ymrN, ymrCRM, ymrEMVI, and ymrTRG), and pathologic variables (i.e., ypT, ypN, ypCRM, and ypTRG). P < 0.05 was considered statistically significant. Statistical analysis was performed using R ver. 3.4.3 (R Foundation for Statistical Computing, Vienna, Austria). 
Table 2. Univariable and multivariable analysis of pathologic circumferential resection margin (CRM) invasion after surgery

\begin{tabular}{|c|c|c|c|c|c|c|}
\hline \multirow{2}{*}{ Variable } & \multicolumn{2}{|c|}{ Univariate analysis } & \multirow{2}{*}{ P-value } & \multicolumn{2}{|c|}{ Multivariate analysis } & \multirow{2}{*}{ P-value } \\
\hline & Pathologic CRM not involved $(n=84)$ & Pathologic CRM involved $(n=28)$ & & $\mathrm{OR}$ & $95 \% \mathrm{Cl}$ & \\
\hline Age (yr) & $65.1 \pm 10.6$ & $62.1 \pm 11.2$ & 0.222 & & & \\
\hline Sex & & & 1.000 & & & \\
\hline Male & $60(71.4)$ & $20(71.4)$ & & & & \\
\hline Female & $24(28.6)$ & $8(28.6)$ & & & & \\
\hline Body mass index $\left(\mathrm{kg} / \mathrm{m}^{2}\right)$ & $22.8 \pm 2.8$ & $22.4 \pm 3.2$ & 0.536 & & & \\
\hline Hemoglobin (g/dL) & $12.9 \pm 2.2$ & $12.7 \pm 2.5$ & 0.127 & & & \\
\hline Tumor location & & & 0.178 & & & \\
\hline Low & $24(28.6)$ & $11(39.3)$ & & & & \\
\hline Mid/upper & $60(71.4)$ & $17(60.7)$ & & & & \\
\hline Tumor size at after CRT (cm) & & & 0.512 & & & \\
\hline$\leq 5$ & $47(56.0)$ & $13(46.4)$ & & & & \\
\hline$>5$ & $37(44.0)$ & $15(53.6)$ & & & & \\
\hline CEA after CRT (ng/mL) & & & 0.038 & & & 0.134 \\
\hline$\leq 5$ & $24(28.6)$ & $2(7.1)$ & & 1.000 & - & \\
\hline$>5$ & $60(71.4)$ & $26(92.9)$ & & 3.557 & $0.795-25.669$ & \\
\hline ymrT category & & & 0.005 & & & 0.164 \\
\hline $0-2$ & 77 (91.7) & $19(67.9)$ & & 1.000 & - & \\
\hline $3-4$ & 7 (8.3) & $9(32.1)$ & & 2.859 & $0.662-13.314$ & \\
\hline ymrN category & & & 0.021 & & & 0.469 \\
\hline Negative & $44(52.4)$ & $7(25.0)$ & & 1.000 & - & \\
\hline positive & $40(47.6)$ & $21(75.0)$ & & 1.539 & $0.480-5.097$ & \\
\hline ymr EMVI & & & 0.002 & & & 0.524 \\
\hline No & $56(66.7)$ & $9(32.1)$ & & 1.000 & - & \\
\hline Yes & 28 (33.3) & $19(67.9)$ & & 1.501 & $0.429-5.373$ & \\
\hline Differentiation & & & 0.381 & & & \\
\hline Well & $50(59.5)$ & $13(46.4)$ & & & & \\
\hline Moderately & $32(38.1)$ & $14(50.0)$ & & & & \\
\hline Poorly & $2(2.4)$ & 1 (3.6) & & & & \\
\hline ymr CRM & & & $<0.001$ & & & 0.025 \\
\hline Converted & 38 (45.2) & $2(7.1)$ & & 1.000 & - & \\
\hline Not converted & $46(54.8)$ & $26(92.9)$ & & 7.030 & $1.497-52.515$ & \\
\hline Surgery type & & & 0.018 & & & \\
\hline LAR & $46(54.8)$ & $7(25.0)$ & & 1.000 & - & \\
\hline ISR & $30(35.7)$ & 15 (53.6) & & 5.748 & $1.765-21.801$ & 0.006 \\
\hline APR & $7(8.3)$ & $6(21.4)$ & & 6.164 & $1.219-35.071$ & 0.031 \\
\hline mrTRG & & & 0.036 & & & \\
\hline $0-2$ & $20(23.8)$ & $2(7.1)$ & & 1.000 & - & 0.711 \\
\hline $3 / 4$ & $64(76.2)$ & $26(92.9)$ & & 1.557 & $0.190-33.111$ & \\
\hline
\end{tabular}

Values are presented as mean \pm standard deviation or number (\%).

CRT, chemoradiotherapy; CEA, carcinoembryonic antigen; EMVI, extramural venous invasion; OR, odds ratio; Cl, confidence interval; LAR, low anterior resection; ISR, intersphincteric resection; APR, abdominoperineal resection; mrTRG, magnetic resonance imaging tumor regression grade. 
Table 3. Univariate analysis for local recurrence-free survival (LRFS) and disease-free survival (DFS)

\begin{tabular}{|c|c|c|c|c|}
\hline \multirow{2}{*}{ Variable } & \multicolumn{2}{|c|}{ LRFS } & \multicolumn{2}{|c|}{ DFS } \\
\hline & $\mathrm{HR}(95 \% \mathrm{Cl})$ & P-value & $\mathrm{HR}(95 \% \mathrm{Cl})$ & P-value \\
\hline \multicolumn{5}{|l|}{ Before CRT } \\
\hline \multicolumn{5}{|l|}{ Age (yr) } \\
\hline$\leq 65$ vs. $>65$ & $1.008(0.453-2.246)$ & 0.983 & $1.071(0.628-1.827)$ & 0.799 \\
\hline \multicolumn{5}{|l|}{ Sex } \\
\hline Male vs. female & $1.862(0.635-5.455)$ & 0.226 & $1.263(0.709-2.246)$ & 0.433 \\
\hline \multicolumn{5}{|l|}{ Body mass index $\left(\mathrm{kg} / \mathrm{m}^{2}\right)$} \\
\hline$\leq 25$ vs. $>25$ & $4.770(0.644-35.355)$ & 0.090 & $1.176(0.574-2.408)$ & 0.651 \\
\hline \multicolumn{5}{|l|}{ Tumor location } \\
\hline Low vs. mid/upper & $2.595(1.159-5.806)$ & 0.023 & $1.379(0.787-2.418)$ & 0.269 \\
\hline \multicolumn{5}{|l|}{ Tumor size $(\mathrm{cm})$} \\
\hline$>5$ vs. $\leq 5$ & $2.361(1.056-5.277)$ & 0.039 & $1.554(0.893-2.704)$ & 0.126 \\
\hline \multicolumn{5}{|l|}{ CEA at diagnosis (ng/mL) } \\
\hline$>5$ vs. $\leq 5$ & $1.831(0.819-4.089)$ & 0.140 & $1.499(0.878-2.558)$ & 0.139 \\
\hline \multicolumn{5}{|l|}{ mrT category } \\
\hline 4 vs. $2 / 3$ & $3.620(1.585-8.253)$ & 0.003 & $2.207(-1.238-3.933)$ & 0.010 \\
\hline \multicolumn{5}{|l|}{ mrN category } \\
\hline N+ vs. NO & $3.584(0.484-26.543)$ & 0.125 & 2.595 (0.809-8.318) & 0.063 \\
\hline \multicolumn{5}{|l|}{ mrEMVI } \\
\hline Yes vs. no & $9.003(1.211-66.901$ & 0.002 & 7.026 (2.184-22.593) & 0.001 \\
\hline \multicolumn{5}{|l|}{ Differentiation } \\
\hline WD vs. MD/PD & $1.228(0.550-2.743)$ & 0.617 & 1.481 (0.867-2.525) & 0.151 \\
\hline \multicolumn{5}{|l|}{ After CRT } \\
\hline \multicolumn{5}{|l|}{ Tumor size at after CRT (cm) } \\
\hline$>5$ vs. $\leq 5$ & $3.952(1.727-9.047)$ & $<0.001$ & $1.554(0.893-2.704)$ & 0.126 \\
\hline \multicolumn{5}{|l|}{ CEA after CRT (ng/mL) } \\
\hline$>5$ vs. $\leq 5$ & $2.749(1.173-6.446)$ & 0.027 & $2.359(1.065-5.223)$ & 0.019 \\
\hline \multicolumn{5}{|l|}{ ymrT category } \\
\hline 4 vs. $2 / 3$ & $5.323(2.270-12.483)$ & $<0.001$ & 2.576 (1.339-4.958) & 0.009 \\
\hline \multicolumn{5}{|l|}{ ymrN category } \\
\hline $\mathrm{N}+$ vs. NO & 2.603 (1.078-6.284) & 0.025 & $1.510(0.880-2.590)$ & 0.140 \\
\hline \multicolumn{5}{|l|}{ ymrEMVI } \\
\hline Yes vs. No & 3.456 (1.476-8.091) & 0.003 & 2.665 (1.543-4.603) & $<0.001$ \\
\hline \multicolumn{5}{|l|}{ ymrCRM } \\
\hline Not converted vs. converted & $3.471(1.184-10.170)$ & 0.010 & 2.537 (1.332-4.833) & 0.002 \\
\hline \multicolumn{5}{|l|}{$\operatorname{mrTRG}$} \\
\hline $0-2$ vs. 3/4 & $6.321(1.010-46.757)$ & 0.015 & $2.720(1.083-6.831)$ & 0.014 \\
\hline \multicolumn{5}{|l|}{ Pathologic variable } \\
\hline \multicolumn{5}{|l|}{ Surgery type } \\
\hline SSS vs. SPS & $2.716(1.071-6.884)$ & 0.054 & $1.538(0.723-3.271)$ & 0.287 \\
\hline
\end{tabular}


Table 3. Continued

\begin{tabular}{|c|c|c|c|c|}
\hline \multirow{2}{*}{ Variable } & \multicolumn{2}{|c|}{ LRFS } & \multicolumn{2}{|c|}{ DFS } \\
\hline & $\mathrm{HR}(95 \% \mathrm{Cl})$ & P-value & $\mathrm{HR}(95 \% \mathrm{Cl})$ & P-value \\
\hline \multicolumn{5}{|l|}{ Lymphovascular invasion } \\
\hline Yes vs. no & 2.397 (0.949-6.056) & 0.088 & $1.435(0.700-2.941)$ & 0.343 \\
\hline \multicolumn{5}{|l|}{ Perineural invasion } \\
\hline Yes vs. no & 3.032 (1.345-6.835) & 0.007 & $1.510(0.880-2.590)$ & 0.140 \\
\hline \multicolumn{5}{|l|}{ Pathologic CRM } \\
\hline Involved vs. not involved & $10.325(4.258-25.033)$ & $<0.001$ & 3.472 (2.020-5.965) & $<0.001$ \\
\hline \multicolumn{5}{|l|}{ Pathologic T } \\
\hline $3 / 4$ vs. $0-2$ & $2.864(0.672-12.210)$ & 0.100 & 3.437 (1.239-9.534) & 0.004 \\
\hline \multicolumn{5}{|l|}{ Pathologic N } \\
\hline Yes vs. No & 2.608 (1.114-6.103) & 0.022 & $2.175(1.225-4.844)$ & 0.015 \\
\hline \multicolumn{5}{|l|}{ Distal margin (cm) } \\
\hline$<1$ vs. $\geq 1$ & $0.864(0.257-2.902)$ & 0.810 & $1.037(0.467-2.301)$ & 0.928 \\
\hline \multicolumn{5}{|l|}{ Pathologic TRG } \\
\hline $0-2$ vs. $3 / 4$ & 1.516 (0.676-3.399) & 0.314 & $1.001(0.577-1.735)$ & 0.998 \\
\hline
\end{tabular}

CRM, circumferential resection margin; CRT, chemoradiotherapy; CEA, carcinoembryonic antigen; EMVI, extramural venous invasion; HR, hazard ratio; Cl, confidence interval; mrTRG, magnetic resonance imaging tumor regression grade; LAR, low anterior resection; ISR, intersphincteric resection; APR, abdominoperineal resection; WD, well differentiated; MD, moderately differentiated; PD, poorly differentiated; TRG, tumor regression grade.

\section{RESULTS}

The median follow-up period of our cohort of 112 patients was 40.6 months (range, $4.2-107.6$ months). Of the 112 patients considered to have potential mrCRM involvement before pCRT, 40 (35.7\%) had mrCRM conversion. Table 1 shows the demographic characteristics and association between persistent CRM involvement and variables defined before pCRT. Univariate analysis showed baseline MRT $(\mathrm{P}=0.020)$, EMVI $(\mathrm{P}<0.001)$, and tumor location $(\mathrm{P}=0.055)$ were associated with persistent $\mathrm{CRM}$ involvement. Multivariate analysis using logistic regression showed that lower tumor location (odds ratio [OR], 3.927; 95\% CI, 1.492$11.182, \mathrm{P}=0.007)$ and positive EMVI on baseline MRI (OR, 3.319; 95\% CI, 1.146-10.149; $\mathrm{P}=0.030$ ) were significant predictive factors for persistent CRM involvement.

Table 2 summarizes the results of uni- and multivariate analyses of posttreatment MR-based variables based on pathologic CRM involvement. Univariate analysis showed posttreatment CEA $(\mathrm{P}=$ 0.038), posttreatment MR T (ymr) $(\mathrm{P}=0.005)$, ymr N $(\mathrm{P}=0.021)$, ymr EMVI $(\mathrm{P}=0.002)$, persistent $\mathrm{CRM}$ involvement $(\mathrm{P}<0.001)$, surgery type $(\mathrm{P}=0.018)$, and ymr TRG $(\mathrm{P}=0.036)$ were significantly associated with pathologic CRM involvement. Multivariate analysis showed persistent CRM involvement on post-treatment MRI (OR, 7.030; 95\% CI, 1.497-52.515; $\mathrm{P}=0.025$ ), intersphincteric resection (ISR; OR, 5.748; 95\% CI, 1.765-21.801; P = 0.006), and abdominopelvic resection (APR; OR, 6.164; 95\% CI, 219$35.071 ; \mathrm{P}=0.031$ ) were significant predictive factors of pathologic
CRM involvement.

During the follow-up period, the local recurrence was 20 of 72 (27.8\%) and 4 of $40(10.0 \%)$ in the persistent CRM involvement and converted CRM groups, respectively. Distant metastasis was found in 42 of 72 patients (58.9\%) and 12 of 40 patients (30.0\%) in the persistent CRM involvement and converted CRM groups, respectively. Uni- (Table 3) and multivariate (Table 4) analyses were performed to address the prognostic significance of persistent CRM status for LRFS and DFS, as well as other parameters. Of pretreatment variables, baseline positive mrEMVI remained significant for DFS (HR, 6.007; 95\% CI, 1.846-19.546; P < 0.001). For the posttreatment variables, APR $(\mathrm{P}<0.001)$ and ISR $(\mathrm{P}<$ 0.012 ) remained significant for LRFS. In addition, persistent CRM involvement (HR, 4.380; 95\% CI, 1.469-13.058; P = 0.008) showed significance. With regard to DFS, persistent CRM involvement (HR, 1.832; 95\% CI, 1.020-3.702; $\mathrm{P}=0.048)$ and positive ymrEMVI (HR, 2.111; 95\% CI, 1.164-3.826; P = 0.014) were significant. Finally, of the pathologic parameters, pathologic CRM involvement was identified as significant not only for LRFS (HR, 9.580; 95\% CI, 3.458-26.535; P < 0.001), but also for DFS (HR, 2.349; 95\% CI, 1.352-4.068; $\mathrm{P}=0.002)$. ypN was only significant for DFS (HR, 2.292; 95\% CI, 1.290-4.067; P = 0.004). Given the prognostic importance of persistent CRM involvement and positive EMVI, the Kaplan-Meier survival curves based on ymrCRM and ymrEMVI status are shown in Fig. 2. A subgroup analysis of the persistent CRM involvement group showed that the 5-year LRFS and DFS were significantly poor among patients with posi- 


\section{Coloproctology chang Hyun Kim, et al.}
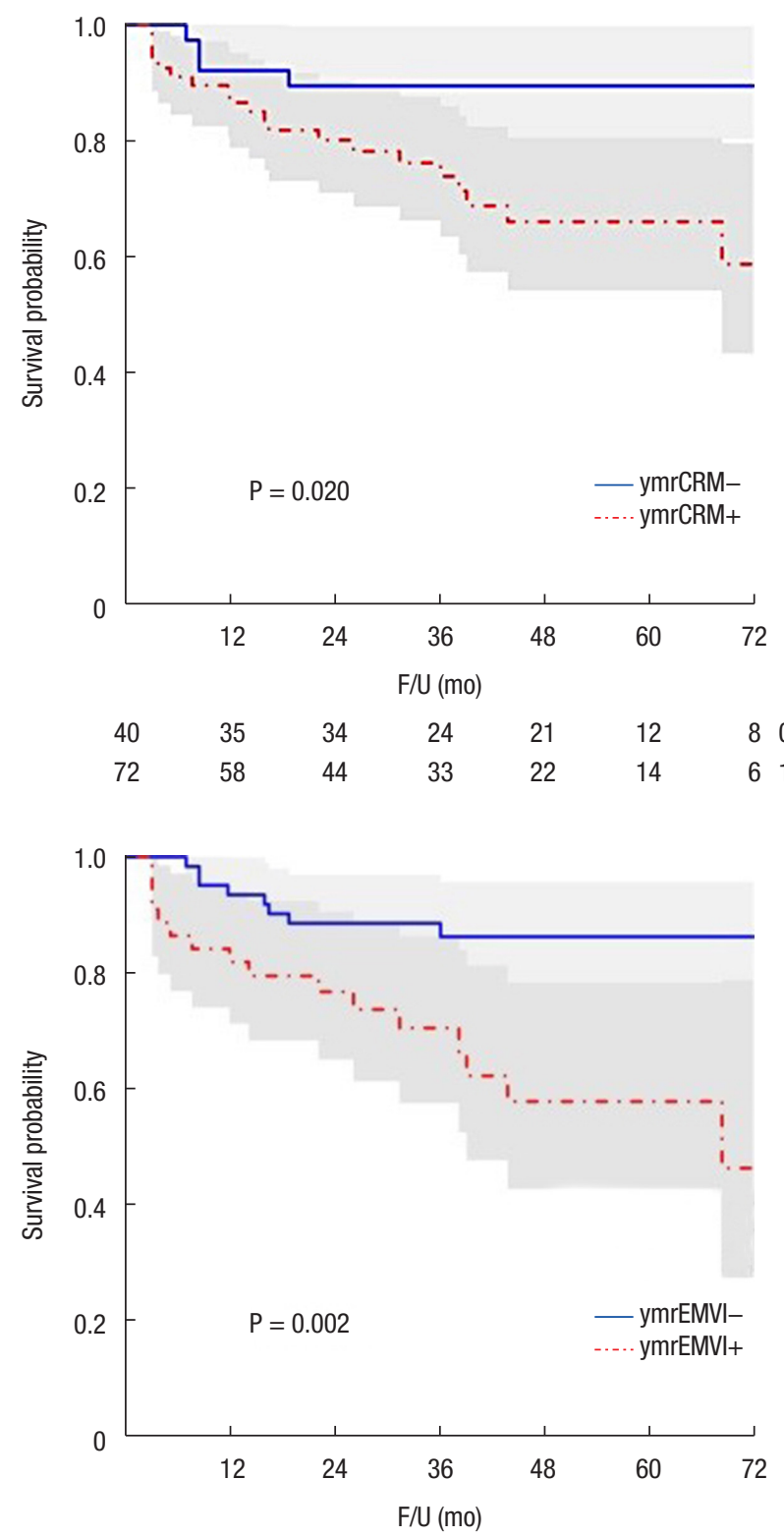
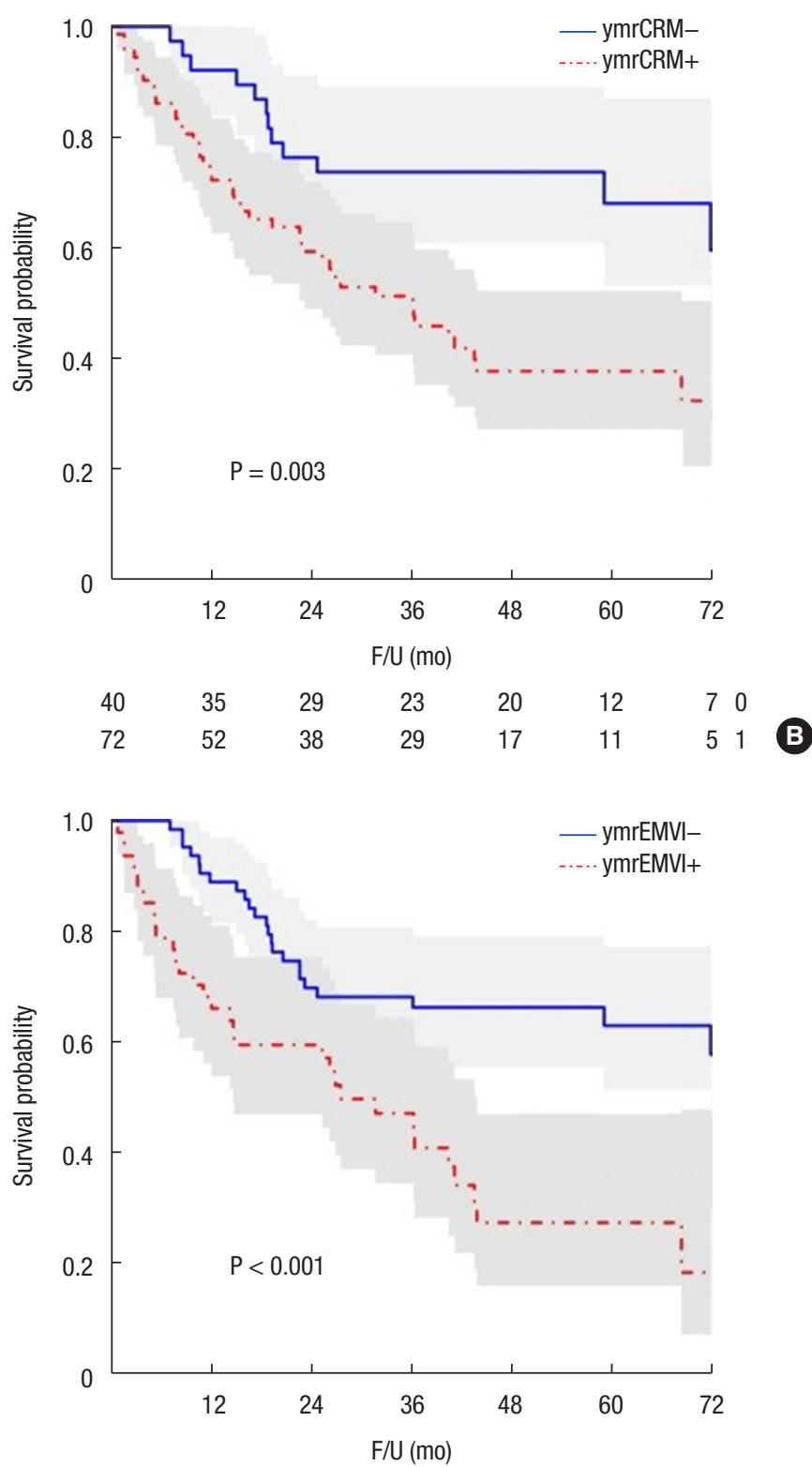

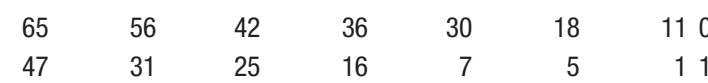

Fig. 2. Survival analysis in entire patients. Local recurrence-free survival (LRFS) (A) and Distant metastasis-free survival (DFS) (B) between positive ymrCRM group and negative ymrCRM group. LRFS (C) and DFS (D) between positive ymrEMVI group and negative ymrEMVI group. ymrCRM, circumferential resection margin evaluated after preoperative chemoradiotherapy (pCRT); ymrEMVI, extramural venous invasion evaluated after pCRT; F/U, follow-up.

tive baseline MR EMVI compared to those who did not have a positive baseline MR EMVI (LRFS [67.9\% vs. 95.6\%, $\mathrm{P}=0.200$ ], DFS [ $36.8 \%$ vs. $87.0 \%, P=0.002]$ ). Similarly, the oncologic outcomes were also significantly poor among patients with positive ymrEMVI (LRFS [57.7\% vs. 86.2\%, P = 0.003], DFS [27.1\% vs. 62.8\%, $\mathrm{P}<0.001]$ ) (Fig. 3).

\section{DISCUSSION}

In this study, the overall CRM conversion rate was $35.7 \%$ in patients with CRM invasion before pCRT. The oncologic outcomes of these patients who did not convert after pCRT were very poor, and positive EMVI on both pre- or posttreatment MRI could fur- 

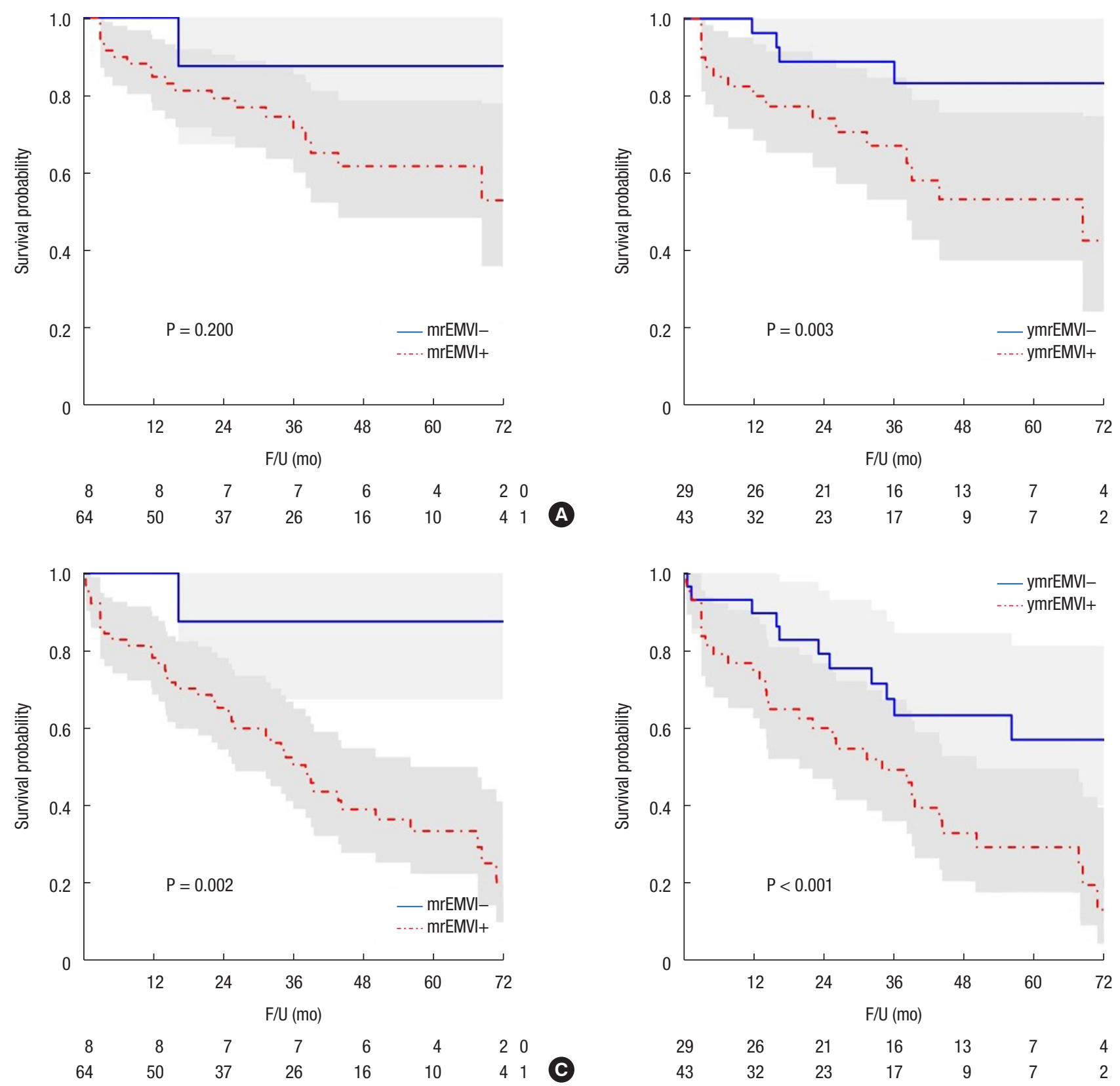

Fig. 3. Survival analysis in persistent circumferential resection margin positive group after preoperative chemoradiotherapy. Local recurrencefree survival (LRFS) according to mrEMVI state (A) and ymrEMVI state (B). Disease-free survival (DFS) according to mrEMVI state (C) and ymrEMVI state (D). mrEMVI, extramural venous invasion evaluated on pretreatment magnetic resonance imaging; ymrEMVI, extramural venous invasion evaluated after preoperative chemoradiotherapy.

ther prognostic information on these patients.

Measurement of CRM involvement in rectal cancer is crucial and well-known as the most powerful prognostic factor for successful surgery [9]. Many studies support the value of CRM involvement not only for LRFS, but also for DFS and overall survival. These results clarified that CRM involvement after pCRT is also important even in patients without pCRT [9]. Although several studies proposed alternative clinical parameters (i.e., mrTRG, tumor volume based on MRI), many studies demonstrate the superiority of CRM $[10,11]$.

The present study evaluates the significance of CRM in LARC requiring more extensive surgery (i.e., beyond the TME plane) af- 


\section{Coloproctology chang Hyun Kim, et al.}

Table 4. Multivariate analysis for local recurrence-free survival (LRFS) and disease-free survival (DFS)

\begin{tabular}{|c|c|c|c|c|}
\hline \multirow{2}{*}{ Variable } & \multicolumn{2}{|c|}{ LRFS } & \multicolumn{2}{|c|}{ DFS } \\
\hline & $\mathrm{HR}(95 \% \mathrm{Cl})$ & P-value & $\mathrm{HR}(95 \% \mathrm{Cl})$ & P-value \\
\hline \multicolumn{5}{|l|}{ Before CRT } \\
\hline \multicolumn{5}{|l|}{ Tumor location } \\
\hline Low vs. mid/upper & 3.205 (1.251-8.209) & 0.015 & & \\
\hline \multicolumn{5}{|l|}{ Tumor size $(\mathrm{cm})$} \\
\hline$>5$ vs. $\leq 5$ & $4.224(1.771-10.078)$ & 0.001 & & \\
\hline \multicolumn{5}{|l|}{ mrT category } \\
\hline 4 vs. $2 / 3$ & $4.762(1.843-12.302)$ & 0.001 & & \\
\hline \multicolumn{5}{|l|}{ mrEMVI } \\
\hline Yes vs. no & & & $6.007(1.846-19.546)$ & $<0.001$ \\
\hline \multicolumn{5}{|l|}{ After CRT } \\
\hline \multicolumn{5}{|l|}{ ymrT category } \\
\hline LAR vs. ISR & $4.303(5.010-59.211)$ & 0.012 & & \\
\hline LAR vs. APR & $17.223(1.782-10.019)$ & $<0.001$ & & \\
\hline \multicolumn{5}{|l|}{ ymrCRM } \\
\hline Not converted vs. converted & 4.380 (1.469-13.058) & 0.008 & $1.832(1.020-3.702)$ & 0.048 \\
\hline \multicolumn{5}{|l|}{ ymrEMVI } \\
\hline Yes vs. no & - & & $2.111(1.164-3.826)$ & 0.014 \\
\hline \multicolumn{5}{|l|}{ Pathologic variables } \\
\hline \multicolumn{5}{|l|}{ Pathologic N } \\
\hline Yes vs. no & & & $2.292(1.290-4.067)$ & 0.004 \\
\hline \multicolumn{5}{|l|}{ Pathologic CRM } \\
\hline Involved vs. not involved & $9.580(3.458-26.535)$ & $<0.001$ & 2.349 (1.352-4.068) & 0.002 \\
\hline
\end{tabular}

CRT, chemoradiotherapy; CEA, carcinoembryonic antigen; EMVI, extramural venous invasion; HR, hazard ratio; Cl, confidence interval; mrTRG, magnetic resonance imaging tumor regression grade; LAR, low anterior resection; ISR, intersphincteric resection; APR, abdominoperineal resection.

ter standard long-course pCRT. The results suggest that pathologic CRM remains the most powerful independent prognostic factor for both LRFS and DFS. Multivariate analysis showed persistent CRM involvement of the posttreatment variable was an independent predictor for pathologic CRM involvement. This result supports the current NCCN guidelines, which regard ymrCRM as a decision tool for deferring surgery and additional chemotherapy. Actually, only 2 of 40 patients (5\%) with converted CRM showed pathologic CRM involvement after surgery. By contrast, in patients showing persistent CRM involvement, ypCRM positive rate was 36\%, and $4(5.5 \%) \mathrm{R} 2$ resections and 3 perforations (4.1\%) were observed. Similarly, Simpson et al. [6] reported that 7 of their 18 patients (38.9\%) with persistent CRM involvement on posttreatment MRI had positive histological margins. In this regard, we can infer that the specificity of posttreatment CRM is low in contrast to its high sensitivity. Therefore, we suggest that surgical exploration should not be abandoned, unless resection is strongly contraindicated, before we obtain firm evidence of a well-performed clinical study. However, surgical planning should include a wider safe margin when surgical resection is attempted in this clinical situation. In our study, ISR and APR were significant predictors of not only pathologic CRM involvement but also poor LRFS. This result is concurrent with that of our previous study that suggested a more radical modification of the standard APR in the treatment of patients with persistent CRM involvement [12].

Furthermore, we identified the factors for prediction of persistent CRM involvement. Tumor location $(\geq 5 \mathrm{~cm})$ and presence of mrEMVI were predictors of persistent CRM involvement after preoperative CRT. This finding is consistent with the previous literature, which reports that negative baseline MRI EMVI (OR, 2.94; $\mathrm{P}=0.007)$, tumor location $\leq 5 \mathrm{~cm}(\mathrm{OR}, 1.96 ; \mathrm{P}=0.02)$, and mrEMVI status change (positive to negative) following pCRT (OR, 3.09; $\mathrm{P}<0.001)$ were the only predictors for response rather than baseline tumor size and tumor stage $[13,14]$. In terms of tumor location, the reason for the good tumor response after pCRT in lower rectal tumors is unclear. A plausible explanation is that relatively fixed anatomical structure in the lower rectum could 
enable more accurate radiotherapy.

EMVI was defined as tumor cell invasion into the surrounding vessels. Concurrent with tumor location, positive mrEMVI (present in $78.5 \%$ of patients) was significantly associated with low CRM conversion rates. This result suggests that this subgroup of patients is resistant to current long-course pCRT and could benefit most from induction chemotherapy $[15,16]$ or additional systemic salvage treatment [4].

In this study, as stated above, unacceptable high ypCRM involvement rate and poor LRFS in patients who did not achieve CRM conversion were shown. In addition to the unsatisfactory local control, poor DFS must also be considered in this group of patients. This result suggests that failure of CRM conversion can be a significant predictor of distant metastasis and local recurrence. Previous studies have shown that positive EMVI is significantly associated with poor 5-year OS, DFS, and LRFS [17]. Our result also showed similar results; positive mrEMVI was associated with a 6-fold increase in the risk of distant metastasis and reduction of 5-year DFS from $87 \%$ to $36.8 \%$ compared with patients without mrEMVI. Strikingly, this poor prognosis is more pronounced in patients with persistent CRM involvement, as shown in Fig. 3. In a subgroup of patients with persistent CRM involvement, the 5-year DFS in patients with mrEMVI and ymrEMVI was $29.8 \%$ and $21.2 \%$, respectively, which is similar to the oncologic outcome for resectable stage IV colorectal cancer [18-20].

This study has potential limitations because of its retrospective nature and relatively small sample size. For example, more details on MR scoring (i.e., mrTRG, meEMVI, both systems use 5-scoring systems) systems were not used and analyzed. However, many strengths also exist, such as largely homogeneous management for a relatively short period of time, high rate of treatment compliance, and to the best of our knowledge, the largest number of patients analyzed.

In conclusion, our study suggests that the current treatment of pCRT and surgery be reconsidered for reaching agreement with NCCN guidelines. Posttreatment mrCRM and EMVI status can be used for decision-making for surgical treatment or salvage systemic treatment, initial baseline EMVI status is a candidate indicator for intensive induction chemotherapy.

\section{CONFLICT OF INTEREST}

No potential conflict of interest relevant to this article was reported.

\section{REFERENCES}

1. Sauer R, Becker H, Hohenberger W, Rödel C, Wittekind C, Fietkau $\mathrm{R}$, et al. Preoperative versus postoperative chemoradiotherapy for rectal cancer. N Engl J Med 2004;351:1731-40.

2. Wiltink LM, Chen TY, Nout RA, Kranenbarg EM, Fiocco M, Laurberg S, et al. Health-related quality of life 14 years after pre- operative short-term radiotherapy and total mesorectal excision for rectal cancer: report of a multicenter randomised trial. Eur J Cancer 2014;50:2390-8.

3. Habr-Gama A, Sabbaga J, Gama-Rodrigues J, São Julião GP, Proscurshim I, Bailão Aguilar P, et al. Watch and wait approach following extended neoadjuvant chemoradiation for distal rectal cancer: are we getting closer to anal cancer management? Dis Colon Rectum 2013;56:1109-17.

4. Sclafani F, Brown G, Cunningham D, Rao S, Tekkis P, Tait D, et al. Systemic Chemotherapy as salvage treatment for locally advanced rectal cancer patients who fail to respond to standard neoadjuvant chemoradiotherapy. Oncologist 2017;22:728-36.

5. Lee NK, Kim CY, Park YJ, Yang DS, Yoon WS, Kim SH, et al. Clinical implication of negative conversion of predicted circumferential resection margin status after preoperative chemoradiotherapy for locally advanced rectal cancer. Eur J Radiol 2014;83: 245-9.

6. Simpson GS, Eardley N, McNicol F, Healey P, Hughes M, Rooney PS. Circumferential resection margin (CRM) positivity after MRI assessment and adjuvant treatment in 189 patients undergoing rectal cancer resection. Int J Colorectal Dis 2014;29:585-90.

7. Kim CH, Lee SY, Kim HR, Kim YJ. Pathologic stage following preoperative chemoradiotherapy underestimates the risk of developing distant metastasis in rectal cancer: a comparison to staging without preoperative chemoradiotherapy. J Surg Oncol 2016;113:692-9.

8. Patel UB, Taylor F, Blomqvist L, George C, Evans H, Tekkis P, et al. Magnetic resonance imaging-detected tumor response for locally advanced rectal cancer predicts survival outcomes: MERCURY experience. J Clin Oncol 2011;29:3753-60.

9. Nagtegaal ID, Quirke P. What is the role for the circumferential margin in the modern treatment of rectal cancer? J Clin Oncol 2008;26:303-12.

10. Glynne-Jones R, Mawdsley S, Pearce T, Buyse M. Alternative clinical end points in rectal cancer--are we getting closer? Ann Oncol 2006;17:1239-48.

11. Glynne-Jones R, Anyamene N. Just how useful an endpoint is complete pathological response after neoadjuvant chemoradiation in rectal cancer? Int J Radiat Oncol Biol Phys 2006;66:31920.

12. Kim CH, Lee SY, Kim HR, Kim YJ. Factors associated with oncologic outcomes following abdominoperineal or intersphincteric resection in patients treated with preoperative chemoradiotherapy: a propensity score analysis. Medicine (Baltimore) 2015;94: e2060.

13. Yu SK, Tait D, Chau I, Brown G. MRI predictive factors for tumor response in rectal cancer following neoadjuvant chemoradiation therapy--implications for induction chemotherapy? Int J Radiat Oncol Biol Phys 2013;87:505-11.

14. Das P, Skibber JM, Rodriguez-Bigas MA, Feig BW, Chang GJ, Wolff RA, et al. Predictors of tumor response and downstaging in patients who receive preoperative chemoradiation for rectal can- 


\section{Coloproctology Chang Hyun Kim, et al.}

cer. Cancer 2007;109:1750-5.

15. Chua YJ, Barbachano Y, Cunningham D, Oates JR, Brown G, Wotherspoon A, et al. Neoadjuvant capecitabine and oxaliplatin before chemoradiotherapy and total mesorectal excision in MRIdefined poor-risk rectal cancer: a phase 2 trial. Lancet Oncol 2010; 11:241-8.

16. Dewdney A, Cunningham D, Tabernero J, Capdevila J, Glimelius B, Cervantes A, et al. Multicenter randomized phase II clinical trial comparing neoadjuvant oxaliplatin, capecitabine, and preoperative radiotherapy with or without cetuximab followed by total mesorectal excision in patients with high-risk rectal cancer (EXPERT-C). J Clin Oncol 2012;30:1620-7.

17. Smith NJ, Barbachano Y, Norman AR, Swift RI, Abulafi AM, Brown G. Prognostic significance of magnetic resonance imag- ing-detected extramural vascular invasion in rectal cancer. $\mathrm{Br}$ J Surg 2008;95:229-36.

18. Kim CH, Huh JW, Kim HR, Kim YJ. Indeterminate pulmonary nodules in colorectal cancer: follow-up guidelines based on a risk predictive model. Ann Surg 2015;261:1145-52.

19. Kim CH, Huh JW, Kim HJ, Lim SW, Song SY, Kim HR, et al. Factors influencing oncological outcomes in patients who develop pulmonary metastases after curative resection of colorectal cancer. Dis Colon Rectum 2012;55:459-64.

20. Bae SU, Han YD, Cho MS, Hur H, Min BS, Baik SH, et al. Oncologic outcomes of colon cancer patients with extraregional lymph node metastasis: comparison of isolated paraaortic lymph node metastasis with resectable liver metastasis. Ann Surg Oncol 2016; 23:1562-8. 